\title{
Effect of combined exercise training on heart rate variability in normotensive and hypertensive postmenopausal women
}

\author{
Igor M. Mariano $^{1}$ (D), Victor Hugo de Freitas $^{1}$ (D), Jaqueline P. Batista ${ }^{1}$ (D), \\ Tállita C.F. de Souza $^{1}$ (D), Ana Luiza Amaral ${ }^{1}$ (D), Juliene G.C. Dechichi ${ }^{1}$ (D), \\ Mateus L. Rodrigues ${ }^{1}$ (D), Victor Hugo V. Carrijo ${ }^{1}$ (D), Guilherme M. Puga ${ }^{1}$ (D) \\ ${ }^{1}$ Universidade Federal de Uberlândia, Faculdade de Educação Física, Laboratório de Fisiologia \\ Cardiorrespiratória e Metabólica, Uberlândia, MG, Brasil.
}

Associate editor: Katia de Angelis, Universidade Federal de São Paulo, Departamento Fisiologia, São Paulo, SP, Brasil.

\begin{abstract}
Aim: This study aimed to verify and compare the effects of 10 weeks of combined exercise training on the heart rate variability of normotensive (NT) and hypertensive (HT) postmenopausal women. Methods: This is a quasiexperimental controlled clinical trial. Therefore, 14 HT and 12 NT postmenopausal women completed 10 weeks of combined exercise training. The exercise protocol consisted of $45 \mathrm{~min}$ of exercise, performed 3 times a week, consisting of $5 \mathrm{~min}$ of warm-up, $20 \mathrm{~min}$ of resistance exercise, and $20 \mathrm{~min}$ of aerobic exercise. Heart rate variability assessments were performed before and after the end of physical training. Results: Heart rate variability was assessed pre- and post-training periods. Mean RR $(\Delta \mathrm{NT}=95 \pm 88 ; \Delta \mathrm{HT}=38 \pm 127)$, $\mathrm{SDNN}(\Delta \mathrm{NT}=9 \pm 13 ; \Delta \mathrm{HT}=3 \pm 14)$, RMSSD $(\Delta \mathrm{NT}=10 \pm 12 ; \Delta \mathrm{HT}=2 \pm 18), \mathrm{SD} 1(\Delta \mathrm{NT}=7 \pm 8 ; \Delta \mathrm{HT}=1 \pm 13)$, and SD2 $(\Delta \mathrm{NT}=10 \pm 18 ; \Delta \mathrm{HT}=4 \pm 17)$ showed improvements after the intervention (time effects $p<0.05$ ). No parameters presented group or interaction effects $(p \geq 0.05)$. Conclusion: In summary, 10 weeks of combined exercise training improved heart rate variability parameters similarly in both NT and HT postmenopausal women. Therefore, combined exercise training may be used to improve autonomic modulation of the heart rate of postmenopausal women, regardless of the presence of hypertension.
\end{abstract}

Keywords autonomic nervous system, aerobic exercise, resistance exercise, blood pressure.

\section{Introduction}

Heart rate variability (HRV) is a non-invasive measurement to evaluate the autonomic modulation of heart rate $(\mathrm{HR})^{1,2}$. Decreased HRV is related to an increased risk of arrhythmia and sudden cardiac death ${ }^{1}$. Although HRV decreases with aging ${ }^{3}$, this effect is pronounced after menopause $e^{4,5}$, when the decreased level of estrogen may interfere with the modulation of cardiovascular autonomic control $^{6}$. Therefore, it is relevant to investigate strategies capable of improving HRV in these women.

The benefits of physical exercise training to improve HRV have been previously reported in meta-analyses ${ }^{7-10}$. In postmenopausal women, the positive effect of isolated aerobic exercises ${ }^{11}$ and combined with resistance exercises training (i.e. combined exercise training; CET) ${ }^{12}$ to improve HRV have already been reported. In addition to the benefits of CET with regard to cardiac autonomic control, this kind of exercise is recommended by the American College of Sports Medicine to maintain and improve cardiovascular and muscular health and functioning of healthy and old adults ${ }^{13,14}$. Furthermore, CET may positively influence systemic inflammation and oxidative stress, bone health, and climacteric symptoms related to being postmenopausal ${ }^{15,16}$. These factors encourage postmenopausal women to include CET as a training strategy in their lives.

In postmenopausal women, the incidence of hypertension is higher compared to men of a similar age and women before menopause ${ }^{17}$. It is part of the risk groups of cardiovascular diseases, which are the main causes of mortality in the world ${ }^{18}$. Previous studies have shown that hypertensive (HT) patients presented worse HRV indices compared to normotensive (NT) subjects, indicating poor cardiac autonomic control ${ }^{12,19}$. The CET, in turn, may improve HRV parameters in HT premenopausal women ${ }^{20}$. However, in HT postmenopausal women, the effect of CET on HRV has not yet been shown. Furthermore, although CET may have a positive effect on the HRV of NT postmenopausal women ${ }^{12}$, studies are necessary to identify if similar benefits could be reported in HT postmenopausal women.

This study aimed to verify the effects of 10 weeks of combined exercise training on the HRV of normotensive and hypertensive postmenopausal women and compare 
the responses between these groups. The hypothesis is that CET would improve HRV parameters in both HT and NT postmenopausal women, with higher improvements in HT subjects. This hypothesis was raised since HT subjects could have had a reduced HRV compared with NT subjects $^{21,22}$, presenting more sensibility to the training.

\section{Methods}

\section{Experimental approach to the problem}

This is a quasi-experimental controlled clinical trial study, in which HRV was monitored in the HT and NT groups before and after 10 weeks of CET. An incremental treadmill test was performed a minimum of $72 \mathrm{~h}$ before the first day of training to identify the intensity of aerobic training. Body mass, height, and body mass index were measured before treadmill testing. Pre-, post-5 weeks, and post-10 weeks of training, participants performed the one maximum repetition test (1RM) to identify the resistance training workload. All tests were performed respecting $48 \mathrm{~h}$ without exercise and a minimum of $48 \mathrm{~h}$ between tests. HRV recording was performed before and after 10 weeks of training, respecting $48 \mathrm{~h}$ without exercise. The study design is presented in Figure 1. The privation of caffeine and alcohol for $24 \mathrm{~h}$ was required for all tests.

\section{Subjects}

A total of 383 postmenopausal women, aged 5070 years, recruited from traditional media (TV, radio, and posters) in 2015 and 2016 agreed to participate, of which 40 fulfilled the inclusion criteria. The entire study was carried out at the Federal University of Uberlândia. So, 26 subjects (14 hypertensive [HT] and 12 normotensives $[\mathrm{NT}]$ ) completed the training (Figure 2). The inclusion criteria were amenorrhea for at least 12 months; body mass index $\leq 30 \mathrm{~kg} / \mathrm{m}^{2}$; ability to engage in treadmill and resistance exercises; no history of diabetes, cancer, or cardiovascular disease (except for hypertension); not using betablockers; no hormone therapy; and non-smokers. This study was approved by the local ethics committee (CAAE: 40622414.9.0000.5152), and all volunteers were informed of the benefits and risks of the investigation prior to signing informed consent agreeing to participate. This research has been conducted in accordance with the principles set forth in the Helsinki Declaration and was registered at
Clinicaltrials.gov (number: NCT03531034). The present study presents secondary data from this registry of which the primary data have already been published ${ }^{23}$.

The International Physical Activity Questionnaire short-form (IPAQ) was used to evaluate the initial level of physical activity of the volunteers. All participants were instructed to maintain their regular eating habits throughout the study. Furthermore, a food intake analysis through 24-h dietary records was applied by nutritionists on three non-consecutive days before and after training. The dietary data analyses were performed using a web-based program (DIETPRO ${ }^{\circledR}$ 5.7i; Minas Gerais, MG, Brazil) and the United States Department of Agriculture food composition table. This analysis demonstrated that there were no significant changes in dietary patterns during the training (data not shown).

\section{Procedures}

Resting blood pressure was monitored through calibrated and validated automatic monitors ${ }^{24}$ (OMRON® HEM-7113, Shimogyo-Ku, Kyoto, Japan) on three nonconsecutive days. At each moment, three measurements of systolic BP (SBP) and diastolic BP (DBP) were performed, and the mean was considered for analysis.

The incremental treadmill test was adapted from Puga et al. ${ }^{25}$. Briefly, all volunteers performed a submaximal incremental test on a treadmill at $5.5 \mathrm{~km} / \mathrm{h}$, and the intensity was increased using treadmill inclination (1\% every $2 \mathrm{~min}$ ) until volunteers reached $85 \%$ of their predicted maximum HR or 18 of perceived exertion using the Borg Scale. Oxygen uptake and carbon dioxide output were recorded during all tests using a gas analyzer (COSMED QUARK CPET gas analyzer, Rome, Italy). The goal of this test was to identify ventilatory thresholds based on ventilatory equivalents.

The intensity of resistance exercise was evaluated and prescribed based on the $1 \mathrm{RM}^{26}$. This test consisted of a warm-up of two sets of the exercise to be performed at intensities around $50 \%$ and $80 \%$ of the subjective estimate of $1 \mathrm{RM}$, with eight and three repetitions, respectively. After this, a maximum of five attempts per exercise was allowed to find the highest workload at which the volunteer could only make one full movement with a 3 -min rest between attempts ${ }^{26}$.

Resting R-R intervals were recorded for $20 \mathrm{~min}$ in the seated position using an HR monitor (POLAR $\AA$

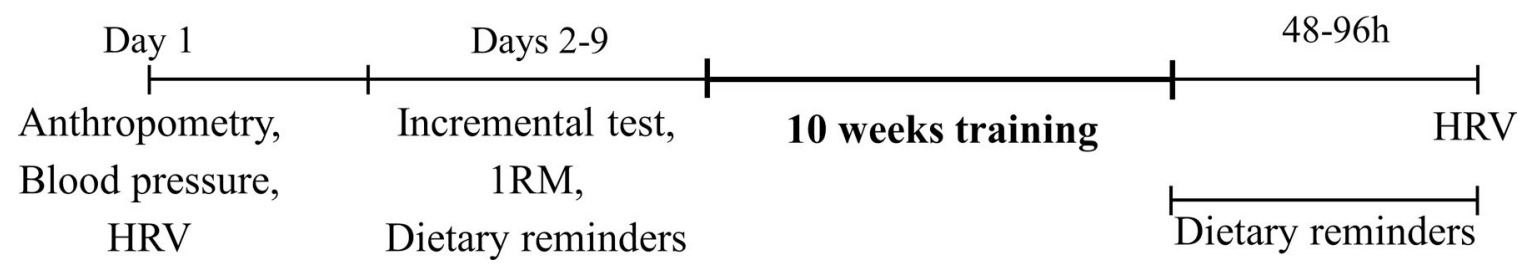

Figure 1 - Study design. HRV: heart rate variability; 1RM: one maximum repetition test. 


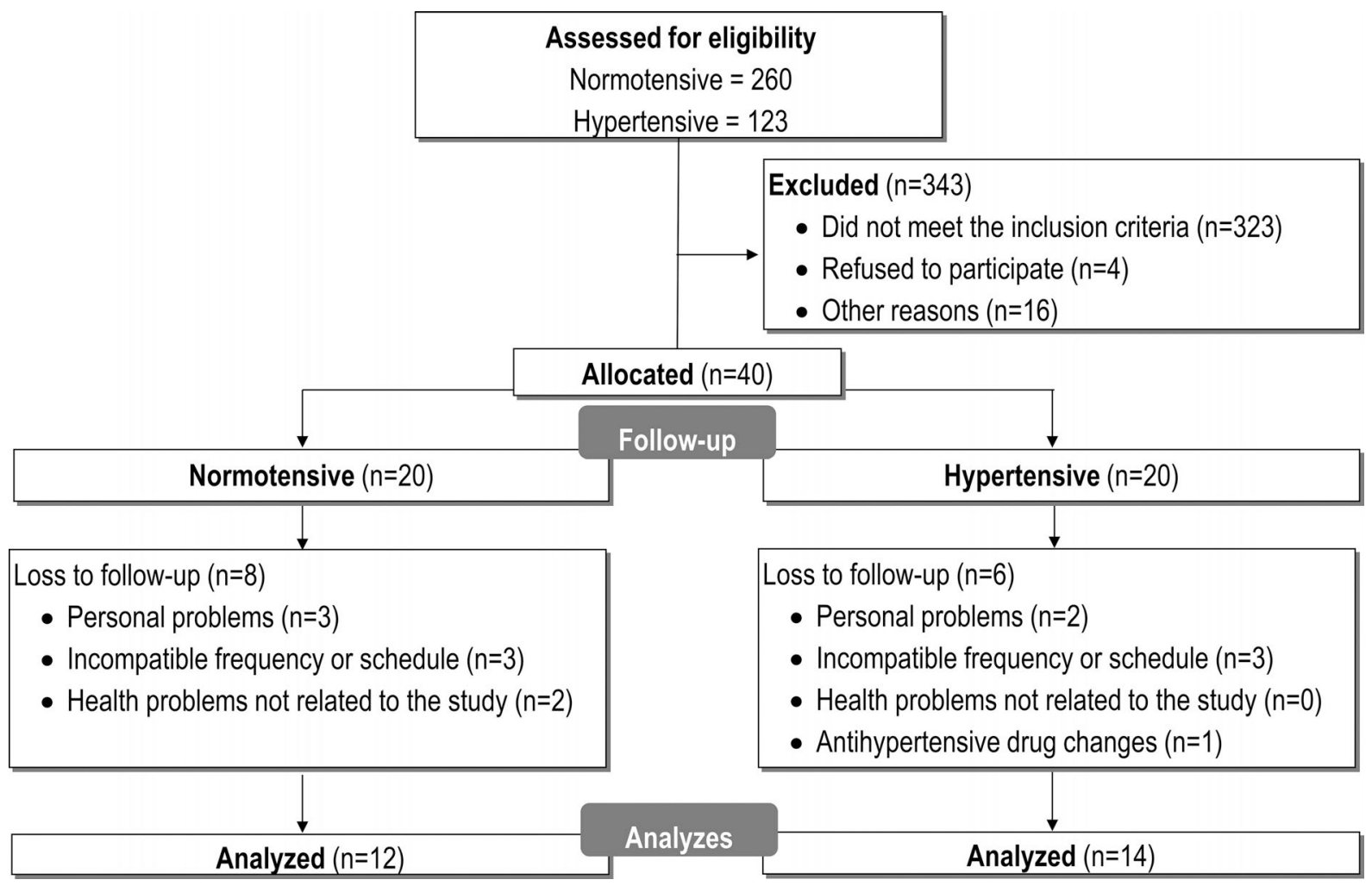

Figure 2 - Follow-up flowchart.

RS800cx; Polar Electro Oy, Finland; sampling frequency $=1000 \mathrm{~Hz}$ ) with spontaneous breathing. Data were downloaded to a computer using an infrared interface with specific software (POLAR PRO TRAINER5®, Polar Electro, Kempele, Finland). HRV analysis was performed using KUBIOS HRV 3.0 (University of Kuopio, Kuopio, Finland $)^{27}$. Prior to the analysis, the signal was visually inspected and filtered, and a range of 5 min with few artifacts was selected close to the end of the recording for analysis.

The resulting R-R intervals were analyzed in the time domain, in the frequency domain using spectral analysis (Fast Fourier Transform), and nonlinearly through the Poincare plot $^{27}$. The time-domain indices analyzed included the square root of the mean squared difference of successive R-R intervals (RMSSD), the standard deviation of all normal R-R intervals recorded at an interval of time (SDNN), and the percentage of pairs of adjacent RRi differing by more than $50 \mathrm{~ms}$ in the whole recording (pNN50). In the frequency domain, the data series were interpolated at $4 \mathrm{~Hz}$, after which removal of the signal linear trend component was performed using the smooth prior approach.

In the frequency domain, oscillations of R-R intervals were examined within the low-frequency (LF: 0.04$0.15 \mathrm{~Hz}$ ) and high-frequency bands (HF: 0.15-0.40 Hz). LF and HF were expressed in normalized units. The sym- pathovagal balance was obtained through the ratio of the LF to HF (LF/HF) bands ${ }^{1}$. For nonlinear indices, the Poincaré plot was examined, and the transversal (SD1) and longitudinal (SD2) axes of the ellipse-like dispersion were calculated.

The exercise program consisted of 30 sessions of combined exercise training performed over 10 consecutive weeks. Each session lasted $45 \mathrm{~min}$ and consisted of $5 \mathrm{~min}$ of warm-up on a treadmill $(5.5 \mathrm{~km} / \mathrm{h}$ and $0 \%$ inclination), $20 \mathrm{~min}$ of resistance exercise, and $20 \mathrm{~min}$ of aerobic exercise. The resistance training was performed in two sets of 15 repetitions at $40 \%$ of $1 \mathrm{RM}$ with 1 min intervals in seven exercises for large muscle groups: leg press $45^{\circ}$, seated low row, vertical chest press, pec deck, wide grip lat pull-down, Swiss ball squat, and abdominal crunch. The aerobic exercise was performed on a treadmill at a velocity of $5.5 \mathrm{~km} / \mathrm{h}$ with an intensity (imposed by the treadmill inclination test reported above) between ventilatory thresholds 1 and 2. After 5 weeks of training, the intensity of the resistance training was adjusted based on a new 1RM, and the intensity of the aerobic exercise was readjusted through a $20 \%$ increase in treadmill inclination.

\section{Statistical analysis}

The sample calculation (minimum $\mathrm{n}=24$ ) was performed in G-Power 3.1 (Universität Düsseldorf, Germany) software $(\alpha$ error $=0.05$ and power $=0.80)$, considering 
RMSSD as the mean variable and $10.3 \pm 17.0 \mathrm{~ms}$ as possible variations in this index after a medium intensity training phase in postmenopausal women ${ }^{20}$. A Cohen's d of 0.6058 was found, which was then transformed into effect size $\mathrm{f}$ for the sample calculation (0.3029). Characteristics and anthropometric values were compared by the t-test for independent samples. Frequencies of physical activity levels were compared using the Chi-square test with the exact Monte Carlo test when the expected count was less than 5 . The normality of data was tested using the Shapiro-Wilk test. A two-factor (time and group) generalized estimating equation technique (GEE) was performed for between, within, and interaction comparisons. Mean RR, LF, HF, and SD2/SD1 presented normality and were analyzed using a linear model. Since some data of pNN50 presented values of 0 , this variable was analyzed using a linear model. Other variables were analyzed using the gamma with log link model. All analyses were performed using IBM ${ }^{\circledR}$ SPSS ${ }^{\circledR}$ Statistics 20 . The significance level adopted was $p<0.05$.

\section{Results}

Table 1 shows the anthropometric, activity level, and drug characteristics of the volunteers. There was a difference only in age, which was higher in the HT group compared with the NT group. HRV parameters (mean and standard deviation) are described in Table 2. Mean RR $(p<0.01), \operatorname{SDNN}(p=0.03), \operatorname{RMSSD}(p=0.03), \operatorname{SD} 1$ $(p=0.03)$, and $\mathrm{SD} 2(p=0.04)$ showed time effects (Table 2). No parameters had group $(p>0.05)$ or interaction $(p>0.05)$ effects.

\section{Discussion}

The present study hypothesized that CET could promote greater improvement in HRV in HT postmenopausal women compared with NT postmenopausal women. Our results refute this hypothesis since we found no differences between NT and HT postmenopausal women in adaptations to CET in mean RR, SDNN, RMSSD, SD1, and SD2.

A greater effect of CET on the HRV of HT postmenopausal women was expected, because the cardiac autonomic modulation of HT subjects at rest was impaired, reflecting in lower general and vagal parameters of $\mathrm{HRV}^{21,22}$. For example, the overall variability measured by SDNN can be up to $15 \%$ lower in HT when compared to healthy ones ${ }^{21}$. Apparently, a trainability effect was expected on HRV, with subjects with lower HRV having a higher effect with training ${ }^{28}$. This improvement can reach up to $50 \%$ of the overall variability measured by SDNN after combined training in women ${ }^{20}$. However, participants of the present study presented well-controlled hypertension (SBP: $121.8 \pm 13.1 \mathrm{~mm} \mathrm{Hg}$; DBP: $76.0 \pm$ $7.8 \mathrm{~mm} \mathrm{Hg}$ ), which may have mitigated the autonomic differences between the HT and NT groups (Table 2). So, the use of antihypertensive drugs may explain why we did not find statistical differences between the groups. However, only the use of atenolol, with or without amlodipine, is related to modifications in HRV at rest in HT patients ${ }^{29}$, which is a family of medicines not used by subjects in the present study. Therefore, additional studies are desired to investigate if antihypertensive drugs may affect rest HRV as well as the effect of exercise training on the HRV of HT postmenopausal women. Up to now, the results suggest no

Table 1 - Anthropometric, activity level, and medical characteristics of normotensive and hypertensive groups. Data are presented in Mean \pm Standard Deviation or frequency (\% within the group).

\begin{tabular}{|c|c|c|c|c|c|c|}
\hline & NT (n=12) & HT $(n=14)$ & $p$ (t test) & & NT $(n=12)$ & HT $(n=14)$ \\
\hline Characteristics & & & & Antihypertensives & & \\
\hline Age (years) & $53.1 \pm 5.3$ & $58.7 \pm 3.8$ & $<0.01$ & ACEi (n) & - & $1(7.1)$ \\
\hline Amenorrhea (years) & $5.0 \pm 3.9$ & $7.2 \pm 6.2$ & 0.30 & ACEi + Diuretic (n) & - & $1(7.1)$ \\
\hline $\mathrm{SBP}(\mathrm{mmHg})$ & $128 \pm 18$ & $122 \pm 13$ & 0.30 & $\mathrm{ARB}(\mathrm{n})$ & - & $4(28.6)$ \\
\hline DBP $(\mathrm{mmHg})$ & $84 \pm 13$ & $76 \pm 8$ & 0.06 & $\mathrm{ARB}+$ Diuretic (n) & - & $5(35.7)$ \\
\hline Anthropometrics & & & & Thiazide Diuretic (n) & - & $3(21.4)$ \\
\hline Height (m) & $1.57 \pm 0.06$ & $1.58 \pm 0.07$ & 0.60 & & & \\
\hline Body Mass (kg) & $64.9 \pm 9.4$ & $69.2 \pm 8.4$ & 0.22 & Other medicines & & \\
\hline BMI $\left(\mathrm{kg} / \mathrm{m}^{2}\right)$ & $26.9 \pm 3.0$ & $27.9 \pm 4.5$ & 0.53 & Calcium (n) & $1(8.3)$ & $3(21.4)$ \\
\hline Physical Activity level & & & $\mathrm{p}\left(\mathrm{X}^{2}\right)$ & Statin (n) & $2(16.6)$ & $3(21.4)$ \\
\hline Sedentary (n) & - & - & 0.33 & Anti-depressant (n) & - & $2(14.3)$ \\
\hline Irregularly active (n) & $7(58.3)$ & $6(42.9)$ & & PPI (n) & - & $1(7.1)$ \\
\hline Active (n) & $4(33.3)$ & $8(57.1)$ & & Beclomethasone (n) & - & $1(7.1)$ \\
\hline Very active (n) & $1(8.3)$ & - & & Levothyroxine (n) & $1(8.3)$ & - \\
\hline
\end{tabular}

NT: Normotensive group; HT: Hypertensive group; SD: Standard deviation; BMI: Body mass index; SBP: Systolic blood pressure; DBP: Diastolic blood pressure; ACEi: Angiotensin-converting enzyme inhibitor; ARB: Angiotensin 1 receiver blockers; PPI: Proton pump inhibitor. 
Table 2 - Heart rate variability parameters on normotensive and hypertensive postmenopausal women.

\begin{tabular}{|c|c|c|c|c|c|c|}
\hline & Groups & Pre $($ mean \pm SD) & Post $($ mean \pm SD) & p Group & p Time & p Interaction \\
\hline \multirow[t]{2}{*}{ Mean RR } & NT & $760.12 \pm 104.59$ & $855.31 \pm 114.19$ & 0.50 & $<0.01$ & 0.16 \\
\hline & HT & $813.44 \pm 132.19$ & $851.33 \pm 87.06$ & & & \\
\hline \multirow[t]{2}{*}{ SDNN } & NT & $20.95 \pm 14.35$ & $29.68 \pm 17.25$ & 0.69 & 0.03 & 0.31 \\
\hline & HT & $21.77 \pm 12.19$ & $24.73 \pm 8.99$ & & & \\
\hline \multirow[t]{2}{*}{ RMSSD } & NT & $15.64 \pm 11.40$ & $25.88 \pm 14.87$ & 0.60 & 0.03 & 0.12 \\
\hline & HT & $21.39 \pm 15.98$ & $23.36 \pm 9.64$ & & & \\
\hline \multirow[t]{2}{*}{ pNN50 } & NT & $2.43 \pm 6.71$ & $8.05 \pm 10.51$ & 0.64 & 0.48 & 0.05 \\
\hline & HT & $7.95 \pm 13.17$ & $5.34 \pm 6.80$ & & & \\
\hline \multirow[t]{2}{*}{ LF } & NT & $73.66 \pm 9.48$ & $67.62 \pm 18.19$ & 0.29 & 0.66 & 0.36 \\
\hline & HT & $65.02 \pm 21.82$ & $67.14 \pm 13.78$ & & & \\
\hline \multirow[t]{2}{*}{$\mathrm{HF}$} & NT & $26.27 \pm 9.47$ & $32.28 \pm 18.12$ & 0.29 & 0.66 & 0.36 \\
\hline & HT & $34.86 \pm 21.78$ & $32.80 \pm 13.78$ & & & \\
\hline \multirow[t]{2}{*}{ SD1 } & NT & $11.09 \pm 8.08$ & $18.35 \pm 10.54$ & 0.60 & 0.03 & 0.12 \\
\hline & HT & $15.15 \pm 11.32$ & $16.55 \pm 6.83$ & & & \\
\hline \multirow[t]{2}{*}{ SD2 } & NT & $27.43 \pm 18.75$ & $37.31 \pm 22.74$ & 0.52 & 0.04 & 0.46 \\
\hline & HT & $26.38 \pm 13.75$ & $30.54 \pm 11.31$ & & & \\
\hline \multirow[t]{2}{*}{$\mathrm{SD} 2 / \mathrm{SD} 1$} & NT & $2.63 \pm 0.63$ & $2.22 \pm 0.78$ & 0.07 & 0.11 & 0.59 \\
\hline & HT & $2.18 \pm 0.85$ & $1.98 \pm 0.56$ & & & \\
\hline
\end{tabular}

NT: Normotensive group; HT: Hypertensive group; SDNN: Standard deviation of normal RR intervals; RMSSD: Root Mean Square of the Successive Differences of RR intervals; pNN50: percentage of pairs of adjacent RR intervals differing by more than 50 ms; LF: Low frequency; HF: High frequency; SD1: Standard deviations of the distances from points to diagonal $\mathrm{Y}=\mathrm{X}$ of the scattergram; SD2: Standard deviations of the distances from points to straight $\mathrm{Y}=-\mathrm{X}+\mathrm{RRmean}$ of the scattergram.

differences that between well-controlled HT and NT postmenopausal women in HRV.

The time effects in the majority of HRV parameters suggest that CET improved the cardiac autonomic control of both NT and HT postmenopausal women. Among these parameters, the RMSSD, pNN50, and SD1 are most affected by high-frequency variations in the HR and are frequently used as a marker of good cardiac vagal modulation $^{1}$. Therefore, the improvement of these parameters suggests that CET increased the resting cardiac vagal modulation of postmenopausal women. These improvements are common physiological adaptations promoted by aerobic training, and an increase in parasympathetic parameters is frequently reported after a phase of training ${ }^{30}$. Improvements in RMSSD and SD1 as a result of CET were previously reported in NT postmenopausal women ${ }^{12}$, corroborating with the results found. However, in accordance with our searches, the improvement in cardiac vagal modulation parameters with CET in HT postmenopausal women is shown for the first time and should be highlighted.

Time effects were reported for mean RR, SDNN, and SD2 too. These parameters are influenced by both low- and high-frequency variations of the HR, therefore, being associated as global parameters of cardiac autonomic control ${ }^{1}$. The positive effect of CET on the mean $\mathrm{RR}$ in NT postmenopausal women was shown pre- viously $^{12}$. These results suggest that, in addition to improvement on cardiac vagal modulation, CET may promote an improvement in the global cardiac autonomic modulation of postmenopausal women. In this population, improvements in autonomic control of the HR are relevant due to the increased risk of cardiovascular diseases associated with low autonomic control of the cardiovascular system $^{3-5,31}$. Studies investigating the effects of CET on HRV in HT postmenopausal women are scarce, making it difficult to compare the results reported here. However, in HT middle-aged sedentary women, CET improved $\mathrm{HRV}^{20}$. In these women, the increase in global HRV is an important clinical effect due to the decreased cardiac autonomic modulation reported in this population ${ }^{2,19}$, with up to $30 \%$ decrease in overall variability as measured by $\mathrm{SDNN}^{4}$.

It is worth mentioning that these results reported in the present study refer to medicated HT postmenopausal women and intervention with combined exercise training with moderate intensity. Therefore, they cannot be generalized to women with untreated or uncontrolled hypertension, men, or exercises with other characteristics. Future studies with a similar design and the presence of a group without antihypertensive drugs could help us to explain the results found. As a possible limitation, we report that there is no group without exercise as an intervention, no control of antihypertensive drug classes and 
doses, and the small sample size that could lead to type 2 error. Finally, we reiterate the importance of physical exercises after menopause regardless of the existence of hypertension, since, besides autonomic control alterations, they can generate improvements in blood pressure ${ }^{23,32,33}$, lipid profile ${ }^{15}$, endothelial function ${ }^{34}$, oxidative profile ${ }^{35}$, climacteric simptoms ${ }^{36}$, and general cardiovascular health $^{33,37}$.

\section{Conclusion}

In summary, 10 weeks of combined exercise training improved the HRV parameters of both normotensive and hypertensive postmenopausal women without significant differences.

\section{Acknowledgments}

This study was supported by the Brazilian government resources through the National Council for Scientific and Technological Development (Grant $n^{\circ}$ 456443/2014-2) and the Minas Gerais State Foundation for Support of Research (Grant n APQ-00750-14).

\section{References}

1. Task Force of the European Society of Cardiology the North American Society of Pacing Electrophysiology. Heart Rate Variability: Standards of Measurement, Physiological Interpretation, and Clinical Use. Circulation 1996;93 (5):104365.

2. Singh JP, Larson MG, Tsuji H, Evans JC, O'Donnell CJ, Levy D. Reduced Heart Rate Variability and New-Onset Hypertension. Hypertension. 1998; 32(2):293-7.

3. Kuo TBJ, Lin T, Yang CCH, Li CL, Chen CF, Chou P. Effect of aging on gender differences in neural control of heart rate. Am J Physiol - Hear Circ Physiol. 1999; 277 (646):2233-9.

4. Brockbank CL, Chatterjee F, Bruce SA, Woledge RC. Heart rate and its variability change after menopause. Exp Physiol. 2000; 85(3):327-30.

5. Neves VFC, Silva de Sá MF, Gallo L, Catai AM, Martins LEB, Crescêncio JC, et al. Autonomic modulation of heart rate of young and postmenopausal women undergoing estrogen therapy. Braz J Med Biol Res. 2007;40 (4):491-9.

6. Mercuro G, Podda A, Pitzalis L, Zoncu S, Mascia M, Melis $\mathrm{GB}$, et al. Evidence of a role of endogenous estrogen in the modulation of the autonomic nervous system. Am J Cardiol. 2000;85(6):787-9.

7. Bhati P, Moiz JA, Menon GR, Hussain ME. Does resistance training modulate cardiac autonomic control? A systematic review and meta-analysis. Clin Auton Res. 2019;29(1):75103.

8. Pearson MJ, Smart NA. Exercise therapy and autonomic function in heart failure patients: a systematic review and meta-analysis. Heart Fail Rev. 2018;23 (1):91-108.
9. Sandercock GRH, Bromley PD, Brodie DA. Effects of exercise on heart rate variability: inferences from a metaanalysis. Med Sci Sports Exerc. 2005;37(3):433-9.

10. Villafaina S, Collado-Mateo D, Fuentes JP, MerellanoNavarro E, Gusi N. Physical Exercise Improves Heart Rate Variability in Patients with Type 2 Diabetes: A Systematic Review. Curr Diab Rep. 2017;17(11): 1-8.0

11. Jurca R, Church TS, Morss GM, Jordan AN, Earnest CP. Eight weeks of moderate-intensity exercise training increases heart rate variability in sedentary postmenopausal women. Am Heart J. 2004;147(5):e8-e15.1

12. Mariano IM, de Freitas VH, Dechichi JGC, Batista JP, de Souza TCF, Amaral AL, et al. Isoflavone does not promote additional effects on heart rate variability of postmenopausal women performing combined exercise training: a clinical, controlled, randomized, double-blind study. Appl Physiol Nutr Metab. 2020;45(4):362-7.2

13. Chodzko-Zajko WJ, Proctor DN, Fiatarone Singh MA, Minson CT, Nigg CR, Salem GJ, et al. American College of Sports Medicine position stand. Exercise and physical activity for older adults. Med Sci Sports Exerc. 2009;41 (7):1510-30.3

14. Garber CE, Blissmer B, Deschenes MR, Franklin BA, Lamonte MJ, Lee IM, et al. American College of Sports Medicine position stand. Quantity and quality of exercise for developing and maintaining cardiorespiratory, musculoskeletal, and neuromotor fitness in apparently healthy adults: Guidance for prescribing exercise. Med Sci Sports Exerc. 2011;43 (7):1334-59.4

15. Giolo JS, Costa JG, da Cunha-Junior JP, Pajuaba ACAM, Taketomi EA, de Souza A V., et al. The effects of isoflavone supplementation plus combined exercise on lipid levels, and inflammatory and oxidative stress markers in postmenopausal women. Nutrients 2018;10 (4):1-11.5

16. Mendoza N, De Teresa C, Cano A, Godoy D, Hita-Contreras F, Lapotka M, et al. Benefits of physical exercise in postmenopausal women. Maturitas 2016;93:83-8.6

17. Di Giosia P, Giorgini P, Stamerra CA, Petrarca M, Ferri C, Sahebkar A. Gender Differences in Epidemiology, Pathophysiology, and Treatment of Hypertension. Curr Atheroscler Rep. 2018;20 (3):13.7

18. Forouzanfar MH, Alexander L, Anderson HR, Bachman VF, Biryukov S, Brauer M, et al. Global, regional, and national comparative risk assessment of 79 behavioural, environmental and occupational, and metabolic risks or clusters of risks in 188 countries, 1990-2013: a systematic analysis for the Global Burden of Disease Study 2013. Lancet. 2015;386(10010):2287-323.8

19. Huikuri H V., Ylitalo A, Pikkujämsä SM, Ikäheimo MJ, Airaksinen KEJ, Rantala AO, et al. Heart rate variability in systemic hypertension. Am J Cardiol. 1996;77(12):1073-7.9

20. Masroor S, Bhati P, Verma S, Khan M, Hussain ME. Heart Rate Variability following Combined Aerobic and Resistance Training in Sedentary Hypertensive Women : A Randomised Control. Indian Heart J. 2018;70(3):S28-S35.0

21. de Andrade PE, do Amaral JAT, Paiva L da S, Adami F, Raimudo JZ, Valenti VE, et al. Reduction of heart rate variability in hypertensive elderly. Blood Press. 2017;26 (6):350-8.1 
22. Mussalo H, Vanninen E, Ikäheimo R, Laitinen T, Laakso M, Länsimies E, et al. Heart rate variability and its determinants in patients with severe or mild essential hypertension. Clin Physiol. 2001;21(5):594-604.2

23. Mariano IM, Dechichi JGC, Matias LAS, Rodrigues M de L, Batista JP, de Souza TCF, et al. Ambulatory blood pressure variability and combined exercise training: comparison between hypertensive and normotensive postmenopausal women. Blood Press Monit. 2020;25:335-45.3

24. Asmar R, Khabouth J, Topouchian J, El Feghali R, Mattar J. Validation of three automatic devices for self-measurement of blood pressure according to the International Protocol: The Omron M3 Intellisense (HEM-7051-E), the Omron M2 Compact (HEM 7102-E), and the Omron R3-I Plus (HEM 6022-E). Blood Press Monit. 2010;15 (1):49-54.4

25. Puga GM, de P Novais I, Katsanos CS, Zanesco A. Combined effects of aerobic exercise and l-arginine ingestion on blood pressure in normotensive postmenopausal women: A crossover study. Life Sci. 2016;151:323-9.5

26. Brown LE, Weir JP. Accurate assessment of muscular strength and power. J Exerc Physiol. 2001;4 (3):1-21.6

27. Tarvainen MP, Niskanen JP, Lipponen JA, Ranta-aho PO, Karjalainen PA. Kubios HRV - Heart rate variability analysis software. Comput. Meth Progr Biomed. 2014;113 (1):210-20.7

28. Soares-Caldeira LF, de Souza EA, de Freitas VH, de Moraes SMF, Leicht AS, Nakamura FY. Effects of Additional Repeated Sprint Training During Preseason on Performance, Heart Rate Variability, and Stress Symptoms in Futsal Players. J Strength Cond Res. 2014;28(10):2815-26.8

29. Pavithran P, Prakash ES, Dutta TK, Madanmohan T. Effect of antihypertensive drug therapy on short-term heart rate variability in newly diagnosed essential hypertension. Clin Exp Pharmacol Physiol. 2010;37(2):e107-e113.9

30. Bellenger CR, Fuller JT, Thomson RL, Davison K, Robertson EY, Buckley JD. Monitoring Athletic Training Status Through Autonomic Heart Rate Regulation: A Systematic Review and Meta-Analysis. Sport. Med. 2016;46(10):146186.0

31. Pathak LA, Shirodkar S, Ruparelia R, Rajebahadur J. Coronary artery disease in women. Indian Heart J. 2017;69 (4):532-8.1
32. Son WM, Sung KD, Bharath LP, Choi KJ, Park SY. Combined exercise training reduces blood pressure, arterial stiffness, and insulin resistance in obese prehypertensive adolescent girls. Clin Exp Hypertens. 2017;39(6):546-52.2

33. Son W-M, Sung K-D, Cho J-M, Park S-Y. Combined exercise reduces arterial stiffness, blood pressure, and blood markers for cardiovascular risk in postmenopausal women with hypertension. Menopause. 2017;24(3):262-8.3

34. Santos-Parker JR, LaRocca TJ, Seals DR. Aerobic exercise and other healthy lifestyle factors that influence vascular aging. Adv Physiol Educ. 2014;38(4):296-307.4

35. Batista JP, Mariano IM, Souza TCF, Costa JG, Giolo JS, Cheik NC, et al. The Acute Effects of Mat Pilates on Hemodynamic and Salivary Nitrate Responses After Exercise in Postmenopausal Women. Int $\mathbf{J}$ Sport Nutr Exerc Metab. 2018;26 (1):1-44.5

36. Costa JG, Giolo JS, Mariano IM, Batista JP, Ribeiro ALA, Souza TCF, et al. Combined exercise training reduces climacteric symptoms without the additive effects of isoflavone supplementation: A clinical, controlled, randomised, double-blind study. Nutr Health. 2017;23(4):271-9.6

37. Lin Y-Y, Lee S-D. Cardiovascular Benefits of Exercise Training in Postmenopausal Hypertension. Int J Mol Sci. 2018;19(9):2523.7

\section{Corresponding author}

Igor Moraes Mariano. Universidade Federal de Uberlândia, Faculdade de Educação Física, Rua Benjamin Constant 1286, Uberlândia, MG, Brasil.

E-mail: igormmariano@gmail.com.

Manuscript received on November 3, 2020

Manuscript accepted on March 29, 2021

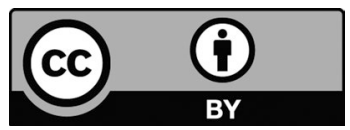

Motriz. The Journal of Physical Education. UNESP. Rio Claro, SP, Brazil - eISSN: 1980-6574 - under a license Creative Commons - Version 4.0 